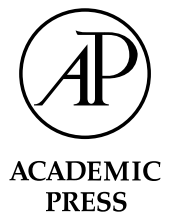

\title{
Role of the adaptor protein CIKS in the activation of the IKK complex
}

\author{
Claudio Mauro, ${ }^{\mathrm{a}}$ Pasquale Vito, ${ }^{\mathrm{b}}$ Stefano Mellone, ${ }^{\mathrm{c}}$ Francesco Pacifico, ${ }^{\mathrm{c}}$ Alain Chariot, ${ }^{\mathrm{d}}$ \\ Silvestro Formisano, ${ }^{a}$ and Antonio Leonardi ${ }^{\mathrm{a}, *}$ \\ a Dipartimento di Biologia e Patologia Cellulare e Molecolare, 'Federico II' University of Naples, Via Pansini 5, Naples 80131, Italy \\ b Dipartimento di Scienze Biologiche e Ambientali, Universita' degli Studi del Sannio, Benevento, Italy \\ ${ }^{\mathrm{c}}$ Istituto di Endocrinologia e Oncologia Sperimentale, CNR, Naples, Italy \\ d Laboratory of Medical Chemistry and Human Genetics, Center for Cellular and Molecular Therapy, C.H. U. Sart-Tilman, 4000 Liège, Belgium
}

Received 29 July 2003

\begin{abstract}
Nuclear factor $\kappa \mathrm{B}(\mathrm{NF}-\kappa \mathrm{B})$ plays a pivotal role in numerous cellular processes, including stress response, inflammation, and protection from apoptosis. Therefore, the activity of $\mathrm{NF}-\kappa \mathrm{B}$ needs to be tightly regulated. We have previously identified a novel gene, named CIKS (connection to IאB-kinase and SAPK), able to bind the regulatory sub-unit NEMO/IKK $\gamma$ and to activate NF$\kappa \mathrm{B}$. Here, we demonstrate that CIKS forms homo-oligomers, interacts with NEMO/IKK $\gamma$, and is recruited to the IKK-complex upon cell stimulation. In addition, we identified the regions of CIKS responsible for these functions. We found that the ability of CIKS to oligomerize, and to be recruited to the IKK-complex is not sufficient to activate the NF- $\kappa \mathrm{B}$. In fact, a deletion mutant of CIKS able to oligomerize, to interact with NEMO/IKK $\gamma$, and to be recruited to the IKK-complex does not activate NF- $\mathrm{NB}$, suggesting that CIKS needs a second level of regulation to efficiently activate NF- $\kappa \mathrm{B}$.
\end{abstract}

(C) 2003 Elsevier Inc. All rights reserved.

Keywords: NF-кB; CIKS/Act1; NEMO/IKK $\gamma$; Signal transduction

The NF- $\kappa \mathrm{B}$ proteins are an evolutionary conserved family of transcription factors that regulate the expression of a variety of cellular genes involved in control of apoptosis, immune and inflammatory responses [1,2]. In most cell types NF- $\mathrm{KB}$ is sequestered in the cytoplasm, bound to inhibitors, collectively called IкBs [3]. Various stimuli, including cytokines, pathogens, and pathogenrelated factors, lead to phosphorylation of $\mathrm{I} \kappa \mathrm{B}$ proteins on specific serine residues (Ser 32 and 36 for $I \kappa B \alpha$ ). This phosphorylation marks $\mathrm{I} \kappa \mathrm{Bs}$ for ubiquitination by the SCF E3 ligase and subsequent degradation through a proteosome-dependent pathway [4]. The IкB inhibitors are phosphorylated by kinases residing in a large molecular weight complex $(700-900 \mathrm{kDa})$ called the IкB kinase-complex (IKK-complex). IKK-complexes are composed of two catalytic sub-units, IKK $\alpha$ and $\beta$ [5-9], and a regulatory sub-unit called NEMO/IKK $\gamma[10,11]$. In addition to the $\mathrm{I} \kappa \mathrm{B}$ proteins, it has been recently

\footnotetext{
${ }^{*}$ Corresponding author. Fax: +39-081-770-1016.

E-mail address: leonardi@unina.it (A. Leonardi).
}

demonstrated that IKKs phosphorylate and regulate the processing of the precursors of $\mathrm{p} 50$ and $\mathrm{p} 52$ sub-units, respectively, $\mathrm{p} 105 / \mathrm{NF}-\kappa \mathrm{B} 1$ and $\mathrm{p} 100 / \mathrm{NF}-\kappa \mathrm{B} 2$ [12-14]. Beside these functions, IKKs may also modulate the transcriptional activity of NF- $\kappa \mathrm{B}$ proteins, phosphorylating the transactivation domain of RelA [15].

NEMO/IKK $\gamma$ was originally identified in a genetic complementation assay, as a factor able to restore NF$\kappa \mathrm{B}$ activation in cells unresponsive to a variety of stimuli that normally induce the NF- $\mathrm{kB}$ pathway [10]. NEMO/ IKK $\gamma$ was also identified in biochemical studies as a component of the high molecular weight IKK-complex [11]. NEMO/IKK $\gamma$ contains a leucine zipper and two coiled-coil domains. These motifs are important for its oligomerization, which is critical for activating the IKK kinase activity [16] and for recruitment of upstream signalling mediators. Different proteins have been demonstrated to interact with NEMO/IKK $\gamma$. These include a kinase known as RIP [17], the cellular protein A20 [17], the viral transactivator tax [18-20], the cellular protein CIKS [21], and the adaptor protein TANK [22]. 
Thus NEMO/IKK $\gamma$ can interact with a variety of different regulatory proteins that are important in regulating the activation of the NF- $\mathrm{KB}$ pathway in response to different stimuli. Despite all these evidences the functions and the mechanisms of NEMO/IKK $\gamma$ activation remain to be determined. Particularly, it is still unclear how the IKKs are activated following different stimuli. It has been proposed that oligomerization of NEMO/IKK $\gamma$ is sufficient to activate the kinase activity of the complex. For example, in response to TNF stimulation, RIP may trigger oligomerization of the IKK-complex through oligomerization of NEMO/IKK $\gamma$ [16]. However, other mechanisms may exist to activate the kinase activity of the IKK-complex, such as direct phosphorylation of the IKK $\alpha / \beta$ by other kinases.

Previously, we reported on the identification of a NEMO/IKK $\gamma$-interacting protein identified in a yeast two-hybrid screen using NEMO/IKK $\gamma$ as bait, which we called CIKS [21] (a.k.a. Act-1) [23]. CIKS does not have any known enzymatic activity and contains a helixloop-helix motif at the amino terminus and a coiled-coil at the carboxyl terminus. Forced expression of CIKS activates NF- $\mathrm{KB}$ and JNK/SAPK pathways, suggesting that CIKS may act as an adaptor protein, linking upstream signalling pathways to the $\mathrm{NF}-\mathrm{\kappa B}$ and JNK/ SAPK pathways. Recently, Qian et al. [24] reported that CIKS may be involved in CD40 signalling, at least in epithelial cells. Kanamori et al. [25] reported a potential involvement of CIKS in the IL-1/Toll pathway, by virtue of its ability to interact with TRAF6.

In this study, we used CIKS as a model to investigate the molecular mechanism by which the IKK-complex is activated. Particularly, we demonstrated that CIKS forms oligomers, interacts with NEMO/IKK $\gamma$, and is recruited to the IKK-complex after cell stimulation. In addition we identified the regions of CIKS responsible for these functions. However, a deletion mutant of CIKS able to oligomerize, to interact with NEMO/ IKK $\gamma$, and to be recruited to the IKK-complex does not activate $\mathrm{NF}-\kappa \mathrm{B}$, suggesting that CIKS needs a second level of regulation to efficiently activate $\mathrm{NF}-\kappa \mathrm{B}$.

\section{Materials and methods}

Cell culture and biological reagents. HeLa, HEK293 were maintained in Dulbecco's modified Eagle's medium supplemented with $10 \%$ fetal calf serum, $1 \%(\mathrm{w} / \mathrm{v})$ penicillin/streptomycin, and $1 \%$ glutamine.

We used two anti-CIKS polyclonal antibodies: one was generated in rabbits and was directed against a recombinant peptide encompassing amino acids 190-382 of human CIKS; the other was from Santa Cruz Biotechnologies. The mouse monoclonal IgM to human CD40 (clone 14G7) was from Caltag. Anti-FLAG and anti-FLAG agarose gel were from Sigma. All other antibodies were from Santa Cruz Biotechnologies.

Full-length NEMO/IKK $\gamma$ and full-length CIKS have been previously described [21]. The HA-CIKS $\Delta \mathrm{C} 300$ (aa 1-274), HA-CIKS $\Delta \mathrm{N} 300$ (aa 300-574), and HA-CIKS $\Delta \mathrm{N} 87$ (aa 87-574) were generated by PCR. For yeast experiments CIKS full-length and different CIKS deletion mutants were cloned in-frame with the Gal4 DNA binding domain of the vector pGBKT7 (Clontech) or with the Gal4 activation domain of the vector pGADT7 (Clontech).

Transfection, immunoprecipitation, and luciferase assays. LipofectAMINE-mediated transfections were performed according to the manufacturer's instructions (Life Technologies). For immunoprecipitation of transfected proteins, HeLa cells $\left(2 \times 10^{6}\right)$ or HEK293 $\left(3 \times 10^{6}\right)$ were transiently transfected and $24 \mathrm{~h}$ after transfection cells were lysed in Triton X-100 lysis buffer [20 mM Hepes, pH 7.4, $150 \mathrm{mM}$ $\mathrm{NaCl}, 10 \%$ glycerol, $1 \%$ Triton $\mathrm{X}-100,1 \mathrm{mM} \mathrm{Na} \mathrm{VO}_{4}, 10 \mathrm{mM} \beta-$ glycerophosphate, $5 \mathrm{mM} \mathrm{NaF}$, and "Complete Protease Inhibitor" mixture (Roche Molecular Biochemicals)]. After an additional $15 \mathrm{~min}$ on ice, cell extracts were centrifuged for $20 \mathrm{~min}$ at $14,000 \mathrm{rpm}$ at $4{ }^{\circ} \mathrm{C}$ and supernatants were immunoprecipitated by using anti-HA or antiFLAG antibodies bound to agarose beads. The immunoprecipitates were washed five times with Triton X-100 lysis buffer and subjected to SDS-PAGE.

For immunoprecipitation of column fractions, fractions were incubated overnight with $10 \mu \mathrm{l}$ of anti-FLAG antibodies bound to agarose beads. The immunoprecipitates were washed five times with Triton X-100 lysis buffer and subjected to SDS-PAGE.

For luciferase assay, HeLa $\left(2 \times 10^{5}\right)$ or HEK293 $\left(4 \times 10^{5}\right)$ were seeded in 6-well $(35 \mathrm{~mm})$ plates. After $12 \mathrm{~h}$ cells were transfected with $0.5 \mu \mathrm{g}$ of Ig- $\mathrm{kB}$-luciferase reporter plasmid and various amounts of each expression plasmid. Total amount of transfected DNA was kept constant by supplementing empty expression plasmid as needed. Cell extracts were prepared $24 \mathrm{~h}$ after transfection and reporter gene activity was determined via the luciferase assay system (Promega). Expression of the pRSV- $\beta$ Gal vector $(0.2 \mu \mathrm{g})$ was used to normalize transfection efficiencies.

Gel filtration of cellular extract. HeLa cells were lysed in Triton X100 lysis buffer. Lysates were incubated for $15 \mathrm{~min}$ on ice and clarified by centrifugation at $14,000 \mathrm{rpm}$ for $15 \mathrm{~min}, 4^{\circ} \mathrm{C}$. Supernatants were collected and recentrifuged for $1 \mathrm{~h}$ at $100,000 \mathrm{~g}$ at $4{ }^{\circ} \mathrm{C}$. One milligram of the S-100 extracts $(0.5 \mathrm{ml})$ was loaded onto a Superdex S200 HR (Amersham-Pharmacia Biotech). Proteins were eluted from the column at the flow rate of $0.3 \mathrm{ml} / \mathrm{min}$. Fractions $(0.4 \mathrm{ml})$ were precipitated with $10 \%$ trichloroacetic acid, resuspended in Laemmli's buffer, and analyzed by SDS-PAGE followed by Western blotting for IKK $\alpha$, IKK $\beta$, NEMO/IKK $\gamma$, and CIKS using appropriate antibodies.

Kinase assay. For IKK kinase assay, endogenous IKK $\beta$ was immunoprecipitated from the fractions eluted from the column by using anti-IKK $\beta$ antibodies and the kinase activity was assayed by using GST-ІкB (aa 1-54) as substrate.

\section{Results}

\section{CIKS forms oligomers}

We have previously identified a novel protein we called CIKS by using NEMO/IKK $\gamma$ as bait in a yeast two-hybrid screening [21] (a.k.a. Act1) [23]. The activities of CIKS loosely resemble those of TRAF proteins. In fact, similar to TRAF2, 5 or 6 , overexpression of CIKS activates SAPK/JNK and IKK kinases [21,2630]. The capacity of TRAFs to activate downstream pathways appears to be linked to the ability of these proteins to oligomerize [30]. In order to investigate if a similar molecular mechanism was involved in the ability of CIKS to activate NF- $\kappa \mathrm{B}$, we examined, in yeast, if CIKS was able to form oligomers. Yeast strain AH109 
was transformed with various deletion mutants of CIKS fused to the GAL4DNA-BD in the presence of fulllength CIKS fused to the GAL4AD (Fig. 1A). The ability of the two fusion proteins to interact was judged by growth of the yeast strain AH109 on selective media. It was possible to detect an interaction between CIKSDNABD and CIKS-AD, suggesting that CIKS was able

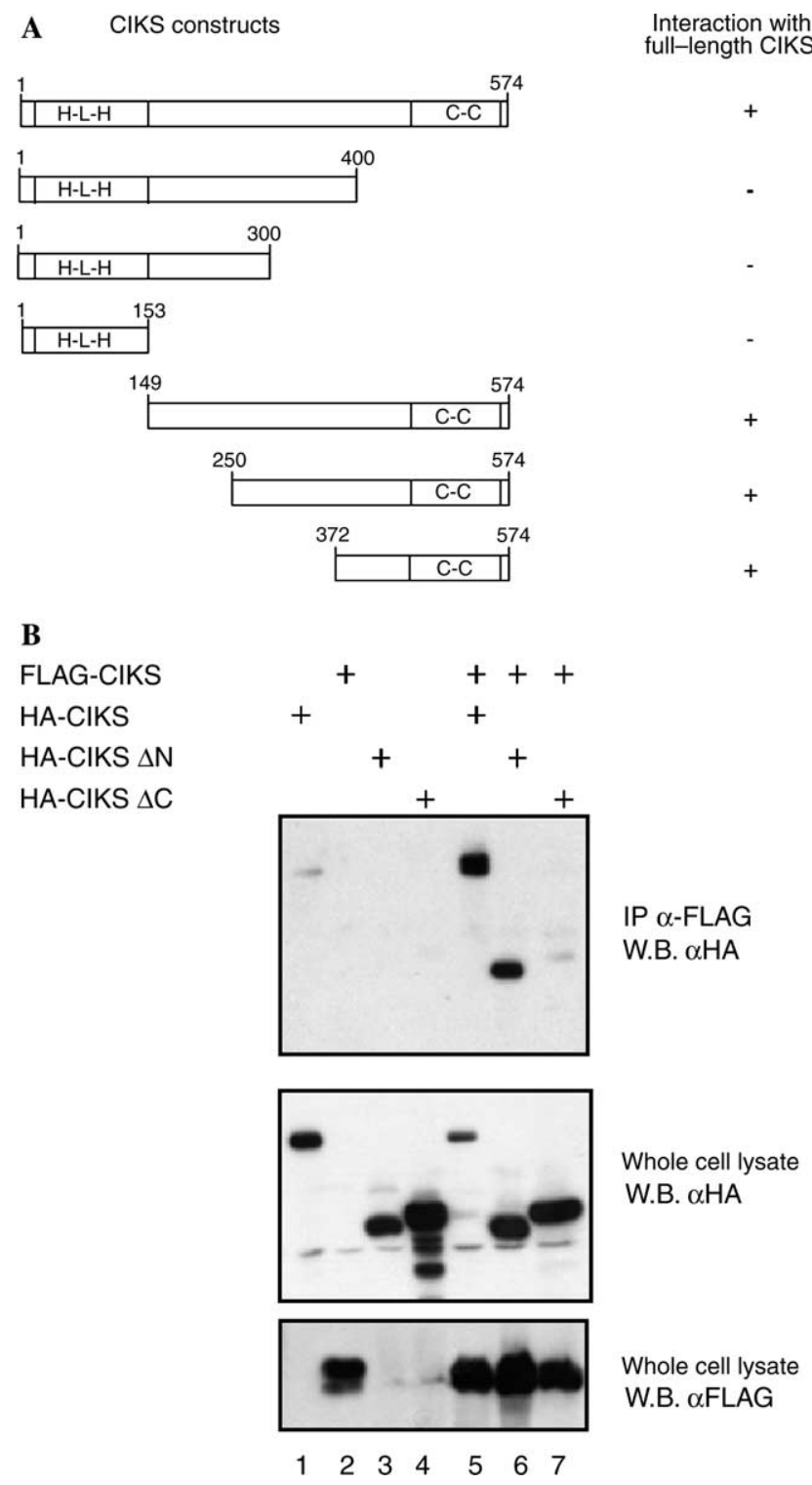

Fig. 1. Mapping the oligomerization domain of CIKS. (A) Yeast-two hybrid experiment. The CIKS constructs cloned in-frame with the GAL4 DNA binding domain of the vector pGBKT7 are schematically illustrated. Plus and minus signs indicate growth or absence of growth of the transformed yeast on medium lacking tryptophan, leucine, histidine, and adenine in the presence of $15 \mathrm{mM}$ of 3-aminotriazole. (B) Co-immunoprecipitation experiment. HeLa cells were transfected with the indicated expression vector. Total extracts were immunoprecipitated with anti-FLAG antibodies followed by Western blot analyses with anti-HA antibodies (top panel). The presence of the different constructs in the whole cell lysate is demonstrated by Western blot in the middle and bottom panels. to oligomerize, at least in yeast. While any deletion at the amino terminus of CIKS did not affect its ability to oligomerize, deletion of the carboxyl-terminus coiledcoil domain disrupted the interaction.

We confirmed the results obtained in yeast, in transient transfection experiments. HeLa cells were transfected with FLAG-tagged CIKS together with HA-tagged CIKS or HA-tagged deletion mutants of CIKS, lacking the amino- or the carboxyl-terminus (CIKS $\Delta$ N300 and CIKS $\Delta$ C300). Cell extracts were immunoprecipitated by using anti-FLAG antibodies and the co-immunoprecipitated proteins were visualized by Western blot with anti-HA-antibodies (Fig. 1B, upper panel). Full- length HA-CIKS and HA-CIKS $\Delta \mathrm{N} 300$ were co-immunoprecipitated with FLAGCIKS (Fig. 1B upper panel, lanes 5 and 6) while HACIKS $\Delta$ C300 was not (Fig. 1B upper panel, lane 7), despite the higher level of expression in HeLa cells. We obtained similar results also in HEK293 cells (data not shown). These data strongly suggest that CIKS is able to form homo-oligomers and that this ability resides in the C-terminal region.

\section{CIKS is recruited to the IKK-complex}

We previously demonstrated that CIKS interacts with NEMO/IKK $\gamma$ and when overexpressed activates the transcription factor NF- $\kappa \mathrm{B}$ via IKKs [21]. Given the ability of CIKS to interact with NEMO/IKK $\gamma$ and to activate $\mathrm{NF}-\kappa \mathrm{B}$, we investigated if CIKS was recruited to the IKK-complex. We reasoned that if forced expression of CIKS mimics the active form of the protein then, when overexpressed, CIKS might be recruited to the IKK-complex. To this purpose, HeLa cells were transiently transfected with an expression vector encoding FLAG-tagged CIKS and cellular extract was loaded onto a Superdex S200 HR Fast Protein Liquid Chromatography column. Fractions eluted from the column were analyzed for the presence of specific components of the IKK-complex and CIKS, using Western blot analysis (Fig. 2). The majority of endogenous IKK $\alpha, \operatorname{IKK} \beta$, and NEMO/IKK $\gamma$ were eluted in a peak centered around a volume of $9 \mathrm{ml}$ (relative molecular mass higher than $660,000 \mathrm{Da}$ ), confirming that the IKKcomplex is preconstituted in unstimulated cells. Analysis of chromatographic distribution of transfected CIKS showed that this protein was eluted in the same fractions containing the components of the IKK-complex. In addition, immunoprecipitating transfected CIKS from these high molecular weight fractions, it was possible to detect endogenous NEMO/IKK $\gamma$ co-immunoprecipitating with transfected CIKS. To further demonstrate that the interaction of CIKS with the IKK-complex correlates with the functional activation of the complex, we immunoprecipitated endogenous IKK $\beta$ from the high molecular weight fractions and assayed its ability 


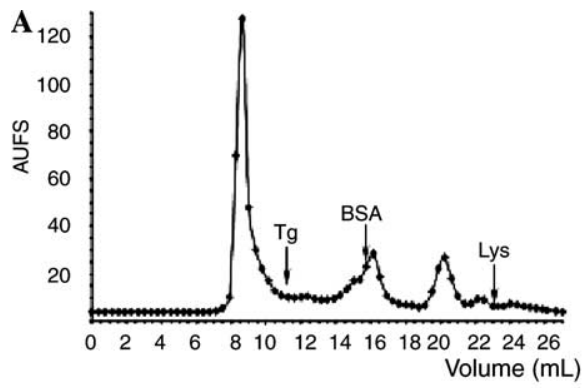

B

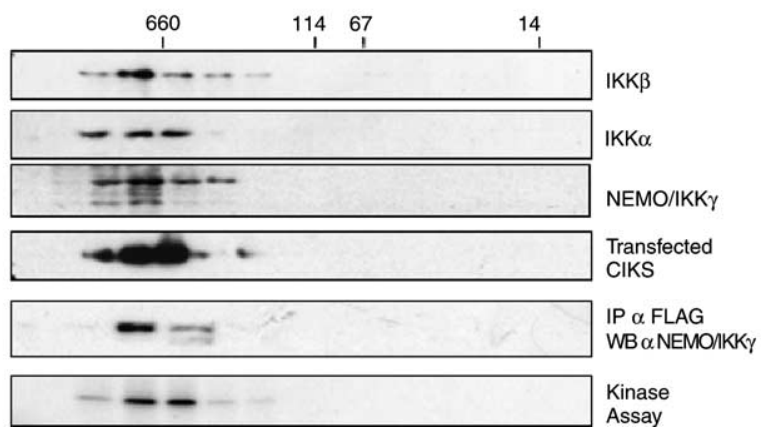

Fig. 2. Chromatographic distribution of CIKS in transfected cells. HeLa cells were transfected with an expression vector encoding FLAG-tagged CIKS. Cell extracts (S-100) were loaded onto a Superdex S200 FPLC column. (A) Elution profile of the column. Position of the molecular weight standards used to calibrate the column is indicated by arrows. Tg: thyroglobulin, MW $660,000 \mathrm{Da}$; BSA: bovine serum albumin, MW 67,000 Da; and Lys: lysozyme, MW 14,000. (B) Fractions isolated from the Superdex S-200 column were subjected to either Western blot or immunoprecipitation or kinase assay. For immunoprecipitation, fractions were incubated with anti-FLAG monoclonal antibodies (clone M2 from Sigma) bound to agarose beads. For kinase assay, fractions were incubated with anti-IKK $\beta$ antibodies (Santa Cruz \#7607). Molecular weight markers are indicated at the top of the figure.

to phosphorylate GST-I $\kappa \mathrm{B} \alpha$. It was possible to isolate a specific IкB kinase activity in high molecular weight fractions from CIKS transfected cells. These data demonstrated that transfected CIKS was recruited to the IKK-complex, via its ability to interact with NEMO/ IKK $\gamma$ and triggered activation of the kinase activity of the complex.

We then analyzed the chromatographic distribution of endogenous CIKS in untransfected cells (Fig. 3). Most of the protein was eluted in a peak centered around a volume of $15 \mathrm{ml}$, corresponding to a molecular mass of about $70,000 \mathrm{Da}$, very similar to its predicted molecular weight of $64,000 \mathrm{Da}$. Recently, it has been reported that CIKS may function as an adaptor molecule in CD40-mediated pathways in epithelial cells [24]. In order to investigate if also the functionally active form of endogenous CIKS was recruited to the IKKcomplex, we analyzed the chromatographic distribution of endogenous CIKS in HeLa cells after CD40 triggering. To this purpose HeLa cells were treated with a stimulating anti-CD40 antibody for thirty minutes. After stimulation, cells were washed and the lysate was

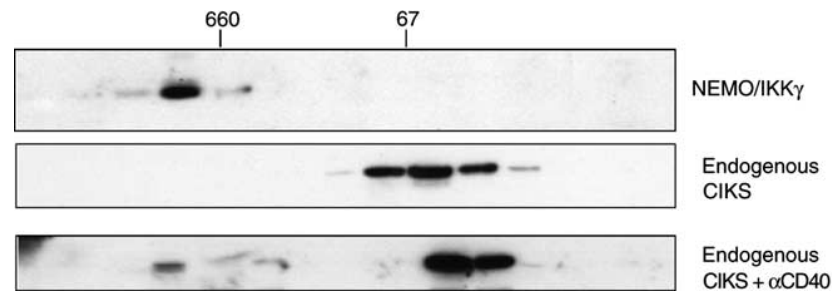

Fig. 3. Chromatographic distribution of endogenous CIKS. HeLa cells were either treated with a stimulating anti-CD40 monoclonal antibody (clone 14G7 from Caltag) for $30 \mathrm{~min}$ or left unstimulated. Cytoplasmic extracts (S100) were prepared and subjected to chromatography on a Superdex S-200 column. Fractions derived from the column were subjected to Western blot by using anti-NEMO/IKK $\gamma$ (Santa Cruz \#8330) or anti-CIKS (Santa Cruz \#11444 or rabbit polyclonal raised against a recombinant CIKS fragment) antibodies.

loaded onto a Superdex S200 HR Fast Protein Liquid Chromatography column. Part of endogenous CIKS was still eluted in a peak corresponding to a molecular mass of 70,000 Da. However, a fraction of CIKS moved to the high molecular weight fractions and was coeluted with components of the IKK-complex (Fig. 3), suggesting that following triggering of CD40, at least part of endogenous CIKS was recruited to the IKK-complex. These results suggested that endogenous CIKS was retained in the cytoplasm in a monomeric form in unstimulated cells, while after stimulation it was recruited to the IKK-complex.

\section{Both the $N$-terminal and the $C$-terminal regions of CIKS are required for interaction with NEMO/IKK $\gamma$}

We next investigated which region of CIKS was necessary for the interaction with NEMO/IKK $\gamma$. HeLa cells were transfected with an expression vector encoding FLAG-tagged NEMO/IKK $\gamma$ in the presence of different HA-tagged CIKS deletion mutants. Cell extracts were immunoprecipitated by using anti-FLAG antibodies and the co-immunoprecipitated proteins were visualized by Western blot with anti-HA antibodies (Fig. 4). Full-length CIKS and the deletion mutant CIKS $\triangle$ N87 were co-immunoprecipitated with FLAGCIKS (Fig. 4 upper panel, lanes 7 and 10). Neither the $\triangle \mathrm{N} 300$ nor the $\Delta \mathrm{C} 300$ mutants interacted with NEMO/ IKK $\gamma$, thus suggesting that the central region of CIKS was involved in the interaction with NEMO/IKK $\gamma$.

CIKS oligomerization, recruitment to the IKK-complex, and interaction with NEMO/IKK $\gamma$ are not sufficient to activate the IKK-complex

One of the proposed mechanisms for activation of the IKK-complex is that forced oligomerization of NEMO/ IKK $\gamma, \operatorname{IKK} \alpha$, and IKK $\beta$ can induce IKK and NF-KB activation [16]. Given the ability of CIKS to form oligomers, to be recruited to the IKK-complex and to 


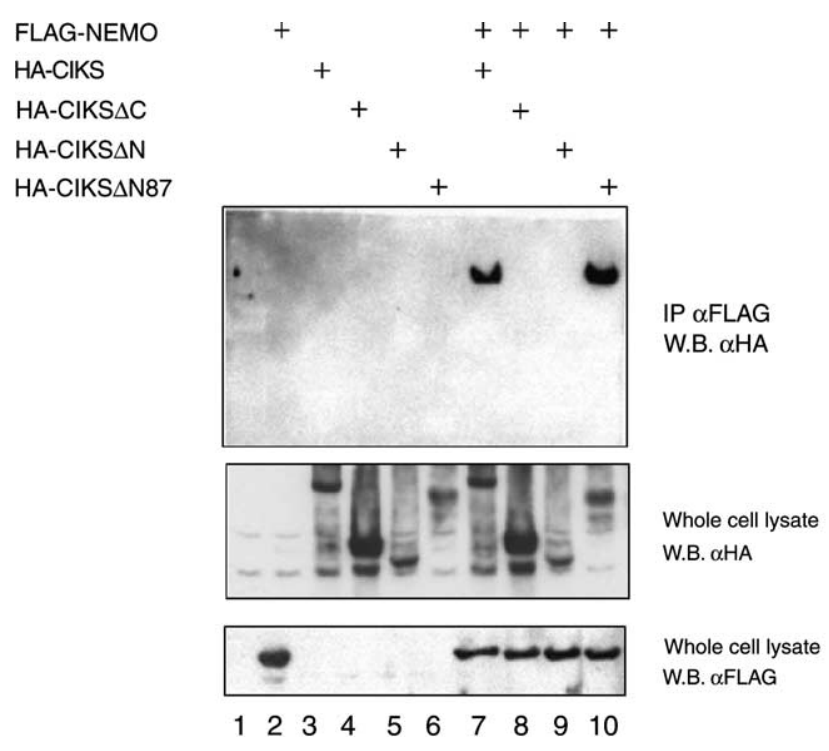

Fig. 4. Mapping the NEMO/IKK $\gamma$ interaction site on CIKS. HeLa cells were transfected with the indicated expression vectors. Total extracts were immunoprecipitated with anti-FLAG antibodies followed by Western blot analyses with anti-HA antibodies (top panel). The presence of the different constructs in the whole cell lysate is demonstrated by Western blot in the middle and bottom panels.

interact with $\mathrm{NEMO/IKK} \gamma$, we investigated which of these properties was necessary to activate NF- $\kappa \mathrm{B}$. HeLa cells were transfected with an Ig- $\kappa \mathrm{B}$ luciferase reporter plasmid in presence of CIKS full-length or different deletion mutants of CIKS (Fig. 5A). Full-length CIKS

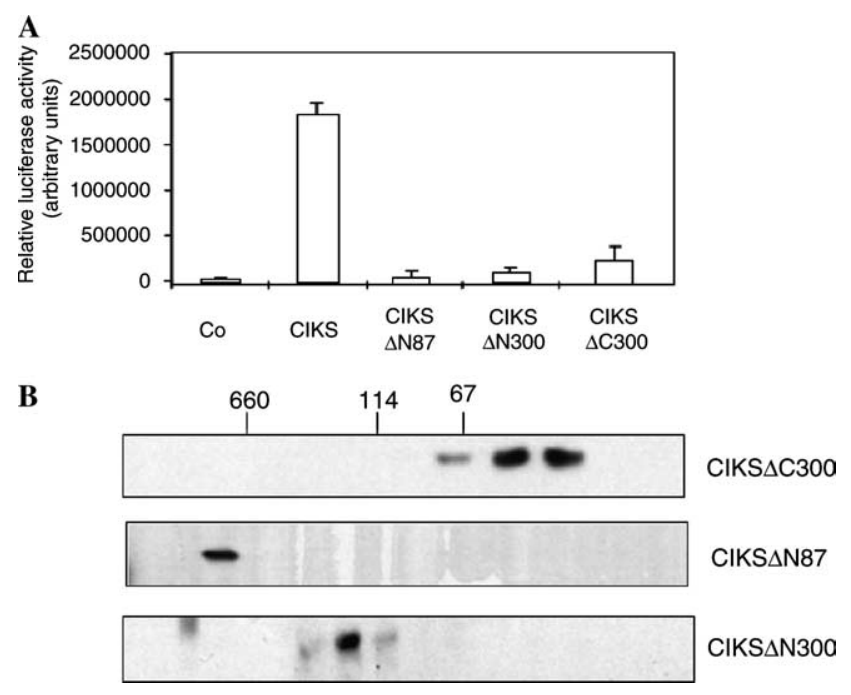

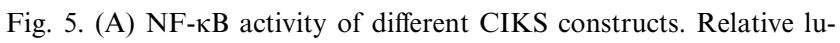
ciferase activity observed in HeLa cells transfected in triplicate with $0.5 \mu \mathrm{g}$ of Ig- $\kappa \mathrm{B}$ luciferase reporter plasmid, with CIKS and different CIKS deletion mutants. Values shown (in arbitrary units) represent means $( \pm \mathrm{SD})$ of three independent experiments, normalized for $\beta$ galactosidase activity of a cotransfected RSV- $\beta$-galactosidase plasmid. (B) Chromatographic distribution of CIKS deletion mutants in transfected cells. HeLa cells were transfected with expression vectors encoding different FLAG-tagged CIKS deletion mutants. Fractions isolated from a Superdex S-200 column were subjected to Western blot analysis by using anti-FLAG antibodies. was able to strongly activate a $\mathrm{\kappa B}$-driven luciferase reporter plasmid, while the $\mathrm{N}$-terminal deletion mutants $\Delta \mathrm{N} 87$ and $\Delta \mathrm{N} 300$ and the C-terminal deletion mutants $\Delta \mathrm{C} 300 \mathrm{did}$ not. The incapacity of the $\Delta \mathrm{N} 300$ and $\Delta \mathrm{C} 300$ mutants to activate NF- $\mathrm{KB}$ might be explained by the inability of these mutants to form oligomers and to interact with NEMO/IKK $\gamma$. Interestingly, the $\Delta \mathrm{N} 87$ deletion mutant that forms oligomers and interacts with NEMO/IKK $\gamma$ does not activate NF- $\kappa \mathrm{B}$. The amount of each transfected plasmid was normalized given the different expression levels of these constructs (see Fig. 1B and data not shown).

Next we investigated the chromatographic distribution of these CIKS mutants. HeLa cells were transfected with the $\Delta \mathrm{N} 87, \Delta \mathrm{N} 300$, and $\Delta \mathrm{C} 300$ deletion mutants. Cell extracts (S100) were loaded onto a Superdex S200 HR Fast Protein Liquid Chromatography column and fractions eluted from the column were analyzed by Western blot by using anti-FLAG antibodies (Fig. 5B). The C-terminal deletion mutant $\Delta \mathrm{C} 300$ was eluted in a peak corresponding to a molecular mass of about $30,000 \mathrm{Da}$. The $\mathrm{N}$-terminus deletion mutants $\Delta \mathrm{N} 87$ and $\Delta \mathrm{N} 300$ were eluted in peaks corresponding to molecular masses higher than $660,000 \mathrm{Da}$ and of about $120,000 \mathrm{Da}$, respectively. The chromatographic distribution of these mutants reflected their ability to form oligomers and to interact with NEMO/IKK $\gamma$. In fact, the $\Delta \mathrm{C} 300$ mutant that was not able to oligomerize and to interact with $\mathrm{NEMO/IKK} \gamma$ was eluted at a volume corresponding to a molecular mass similar to its predicted molecular weight. In contrast, the $\Delta \mathrm{N} 87$ mutant, still able to interact with NEMO/IKK $\gamma$ and to oligomerize, was coeluted with components of the IKK-complex. The $\triangle \mathrm{N} 300$ mutant that retained the ability to oligomerize but not to interact with $\mathrm{NEMO/IKK} \gamma$ was eluted at a volume intermediate between the other two mutants.

Taken together these results suggest that the ability of CIKS to form oligomers and to be recruited to the IKKcomplex resides in different regions of the protein and that both activities are necessary but not sufficient to correctly activate NF-кB.

\section{Discussion}

In the present paper, we reported a functional characterization of the adaptor protein CIKS. We demonstrated that CIKS was able to form oligomers and once overexpressed was recruited to the IKKcomplex through interaction with NEMO/IKK $\gamma$. In addition, we demonstrated that both transfected CIKS and endogenous CIKS after CD40 triggering were recruited to the IKK-complex. The ability of CIKS to form oligomers resided in the $\mathrm{C}$-terminal region, while the ability to interact with NEMO/IKK $\gamma$ was mediated by the central region of the protein. Both the capacities 
to oligomerize and to interact with $\mathrm{NEMO} / \mathrm{IKK} \gamma$ were necessary to activate NF- $\kappa \mathrm{B}$. In fact, deletions affecting one of these functions abolished the ability of CIKS to activate NF- $\kappa \mathrm{B}$. However, these functions were not sufficient for a correct activation of NF- $\mathrm{BB}$. In fact a small $\mathrm{N}$-terminus deletion mutant $(\Delta \mathrm{N} 87)$ still able to interact with $\mathrm{NEMO} / \mathrm{IKK} \gamma$ and to form oligomers did not activate NF- $\kappa \mathrm{B}$. Our data suggest that in addition to oligomerization and interaction with NEMO/IKK $\gamma$, CIKS needs a second level of regulation to efficiently activate $\mathrm{NF}-\kappa \mathrm{B}$.

CIKS has been proposed to interact with TRAF3 and by virtue of this interaction has been proposed to be involved in the CD40 signalling. Quian et al. [24] proposed a role for CIKS in the CD40 pathway. Based on this model CIKS is recruited to CD40 after receptor triggering. Our data extend this model, demonstrating that after CD40 triggering, CIKS connects the receptor and the IKK-complex, through its interaction with $\mathrm{NEMO} / \mathrm{IKK} \gamma$, mediating the activation of NF- $\kappa \mathrm{B}$. It remains to be determined whether the IKK-complex is recruited to the cytoplasmic domain of the receptor or, after interaction with CD40, CIKS dissociates from the receptor to interact with NEMO/IKK $\gamma$.

The functions of CIKS resemble those of TRAF proteins. These are adaptor molecules that mediate NF$\kappa \mathrm{B}$ activation by different receptors, such as members of the TNF and IL-1/Toll receptor family. Activation of $\mathrm{NF}-\kappa \mathrm{B}$ following TNF-receptor engagement is known to be initiated recruiting different adaptor molecules like TRADD, RIP, and TRAF on the trimerized cytoplasmic domain of the TNF receptor [31]. RIP and TRAF, in turn, transiently recruit and activate the IKK complex, via NEMO/IKK $\gamma$ [17,32]. However, it is not yet clear how the IKK-complex is activated. Recently, it has been demonstrated that the enforced oligomerization of $\mathrm{NEMO} / \mathrm{IKK} \gamma$ or any component of the IKK-complex is sufficient to activate the kinase activity of the complex. By virtue of its ability to form oligomers and to interact with NEMO/IKK $\gamma$ after CD40 triggering, CIKS may function in a similar way to activate the IKK-complex. However, while the ability of CIKS to oligomerize and to be recruited to the complex is both necessary to activate $\mathrm{NF}-\mathrm{\kappa B}$, these are not sufficient. In fact, a small deletion of the N-terminal domain of CIKS (CIKS $\Delta \mathrm{N} 87$ ), that leaves unaltered the ability of CIKS to interact with NEMO/IKK $\gamma$, to be recruited to the IKKcomplex and to form oligomers, abolished its ability to activate NF- $\mathrm{KB}$. It is possible that the $\Delta \mathrm{N} 87$ mutant is incapable of inducing conformational changes important for the activation of the kinase activity of the IKKcomplex. However, it is tempting to speculate that the $\mathrm{N}$-terminus of CIKS is interacting with some other adaptor molecule(s) or some kinase(s) that are, in turn, responsible for the activation of the IKK-complex. This hypothesis is currently under investigation. It has been reported previously that CIKS interacts with the protein kinase TAK1 [24] but the exact role of this kinase in CD40-mediated NF- $\kappa \mathrm{B}$ activation needs to be further addressed. In addition, the importance of other MAP3 kinase kinase kinase such as NIK, MEKK1, and MEKK3 in the activation of the IKKs is still controversial.

In summary, our data provide evidences about the role of the adaptor protein CIKS in the activation of the IKK-complex. After CD40 triggering CIKS is recruited to the IKK-complex, through its ability to interact with NEMO/IKK $\gamma$ and to form oligomers. However, these abilities are not sufficient to activate $\mathrm{NF}-\kappa \mathrm{B}$, suggesting that CIKS needs a second level of regulation to efficiently activate NF- $\mathrm{KB}$.

\section{Acknowledgments}

The authors thank R. Acquaviva for assistance with the FPLC and helpful discussion. This work was supported by grants from AIRC (Associazione Italiana Ricerca sul Cancro) to A.L. and Ministero della Universita' e Ricerca Scientifica Grant 2001065217 to S.F.

\section{References}

[1] S. Ghosh, M. Karin, Missing pieces in the NF-kappaB puzzle, Cell (2002) S81-96.

[2] N. Silverman, T. Maniatis, NF-kappaB signaling pathways in mammalian and insect innate immunity, Genes Dev. 15 (2001) $2321-2342$.

[3] I. Verma, J.K. Stevenson, E.M. Schewarz, D. Van Antwerp, S. Miyamoto, Rel/NF-kappa B/I kappa B family: intimate tales of association and dissociation, Genes Dev. 9 (1995) 2723-2735.

[4] M. Karin, Y. Ben-Neriah, Phosphorylation meets ubiquitination: the control of NF-[kappa]B activity, Annu. Rev. Immunol. 18 (2000) 621-663.

[5] J.A. Di Donato, M. Hayakawa, D.M. Rothwarf, E. Zandi, M. Karin, A cytokine-responsive IkappaB kinase that activates the transcription factor NF-kappaB, Nature 388 (1997) 548-554.

[6] F. Mercurio, H. Zhu, B.W. Murray, A. Shevchenko, B.L. Bennett, Y. Li, D.B. Young, M. Barbosa, M. Mann, A. Manning, A. Rao, IKK-1, IKK-2: cytokine-activated IkappaB kinases essential for NF-kappaB activation, Science 278 (1997) 860-866.

[7] C.H. Regnier, H.Y. Song, X. Gao, D.V. Goeddel, Z. Cao, M. Rothe, Identification and characterization of an IkappaB kinase, Cell 90 (1997) 373-383.

[8] J.D. Woronicz, X. Gao, Z. Cao, M. Rothe, D.V. Goeddel, IkappaB kinase-beta: NF-kappaB activation and complex formation with IkappaB kinase-alpha and NIK, Science 278 (1997) 866-869.

[9] E. Zandi, D.M. Rothwarf, M. Delhase, M. Hayakawa, M. Karin, The IkappaB kinase complex (IKK) contains two kinase subunits, IKKalpha, IKKbeta, necessary for IkappaB phosphorylation, NF-kappaB activation, Cell 91 (1997) 243-252.

[10] S. Yamaoka, G. Courtois, C. Bessia, S.T. Whiteside, R. Weil, F. Agou, H.E. Kirk, R.Y. Kai, A. Israel, Complementation cloning of NEMO, a component of the IkappaB kinase complex essential for NF-kappaB activation, Cell 93 (1998) 1231-1240.

[11] D.M. Rothwarf, E. Zandi, G. Natoli, N. Karin, IKK-gamma is an essential regulatory subunit of the IkappaB kinase complex, Nature 395 (1998) 297-300. 
[12] V. Heissmeyer, D. Krappmann, E.N. Hatada, C. Scheidereit, Shared pathways of IkappaB kinase-induced SCF(betaTrCP)mediated ubiquitination and degradation for the NF-kappaB precursor p105, IkappaBalpha, Mol. Cell. Biol. 4 (2001) $1024-1035$.

[13] A. Salmeron, J. Janzen, Y. Soneji, N. Bump, J. Kamens, H. Allen, S.C. Ley, Direct phosphorylation of NF-kappaB1 p105 by the IkappaB kinase complex on serine 927 is essential for signalinduced p105 proteolysis, J. Biol. Chem. 276 (2001) 22215-22222.

[14] U. Senftleben, Y. Cao, G. Xiao, F.R. Greten, G. Krahn, G. Bonizzi, Y. Chen, Y. Hu, A. Fong, S.C. Sun, M. Karin, Activation by IKKalpha of a second, Science 293 (2001) 1495-1499.

[15] H. Sakurai, H. Chiba, H. Miyoshi, T. Sugita, W. Toriumi, IkappaB kinases phosphorylate NF-kappaB p65 subunit on serine 536 in the transactivation domain, J. Biol. Chem. 274 (1999) 30353-30356.

[16] J.L. Poyet, S.M. Srinivasula, J.H. Lin, T. Fernandez-Alnemri, S. Yamaoka, P.N. Tsichlis, E.S. Alnemri, Activation of the Ikappa B kinases by RIP via IKKgamma/NEMO-mediated oligomerization, J. Biol. Chem. 275 (2000) 37966-37977.

[17] S.Q. Zhang, A. Kovalenko, G. Cantarella, D. Wallach, Recruitment of the IKK signalosome to the p55 TNF receptor: RIP, A20 bind to NEMO (IKKgamma) upon receptor stimulation, Immunity 12 (2000) 301-311.

[18] Z.L. Chu, Y.A. Shin, J.M. Yang, J.A. Di Donato, D.W. Ballard, IKKgamma mediates the interaction of cellular IkappaB kinases with the tax transforming protein of human $\mathrm{T}$ cell leukemia virus type 1, J. Biol. Chem. 274 (1999) 15297-15300.

[19] E.W. Harhaj, S.C. Sun, IKKgamma serves as a docking subunit of the IkappaB kinase (IKK) and mediates interaction of IKK with the human T-cell leukemia virus Tax protein, J. Biol. Chem. 274 (1999) 22911-22914.

[20] D.Y. Jin, V. Giordano, K.V. Kibler, H. Nakano, K.T. Jeang, Role of adapter function in oncoprotein-mediated activation of NFkappaB. Human T-cell leukemia virus type I Tax interacts directly with IkappaB kinase gamma, J. Biol. Chem. 274 (1999) $17402-17405$.

[21] A. Leonardi, A. Chariot, E. Claudio, K. Cunningham, U. Siebenlist, CIKS, a connection to Ikappa B kinase and stressactivated protein kinase, Proc. Natl. Acad. Sci. USA 97 (2000) 10494-10499.
[22] A. Chariot, A. Leonardi, J. Muller, M. Bonif, K. Brown, U. Siebenlist, Association of the adaptor TANK with the I kappa B kinase (IKK) regulator NEMO connects IKK complexes with IKK epsilon, TBK1 kinases, J. Biol. Chem. 277 (2002) 37029-37036.

[23] X. Li, M. Commane, H. Nie, X. Hua, M. Chatterjee-Kishore, D. Wald, M. Haag, G.R. Stark, Act1 an NF-kappa B-activating protein, Proc. Natl. Acad. Sci. USA 97 (2000) 10489-10493.

[24] Y. Qian, Z. Zhao, Z. Jiang, X. Li, Role of NF kappa B activator Act1 in CD40-mediated signaling in epithelial cells, Proc. Natl. Acad. Sci. USA 99 (2002) 9386-9391.

[25] M. Kanamori, C. Kai, Y. Hayashizaki, H. Suzuki, NF-kappaB activator Act1 associates with IL-1/Toll pathway adaptor molecule TRAF6, FEBS Lett. 532 (2002) 241-246.

[26] T.K. Ishida, T. Tojo, T. Aoki, N. Kobayashi, T. Ohishi, T. Watanabe, T. Yamamoto, J. Inoue, TRAF5 a novel tumor necrosis factor receptor-associated factor family protein, mediates CD40 signaling, Proc. Natl. Acad. Sci. USA 93 (1996) 9437-9442.

[27] H. Nakano, H. Oshima, W. Chung, L. Williams-Abbott, C.F. Ware, H. Yagita, K. Okumura, TRAF5 an activator of NFkappaB and putative signal transducer for the lymphotoxin-beta receptor, J. Biol. Chem. 271 (1996) 14661-14664.

[28] C. Reinhard, B. Shamoon, V. Shyamala, L.T. Williams, Tumor necrosis factor alpha-induced activation of c-jun $\mathrm{N}$-terminal kinase is mediated by TRAF2, EMBO J. 16 (1997) 1080-1092.

[29] H.Y. Song, C.H. Regnier, C.J. Kirschning, D.V. Goeddel, M. Rothe, Tumor necrosis factor (TNF)-mediated kinase cascades: bifurcation of nuclear factor-kappaB and c-jun N-terminal kinase (JNK/SAPK) pathways at TNF receptor-associated factor 2, Proc. Natl. Acad. Sci. USA 94 (1997) 9792-9796.

[30] V. Baud, Z.G. Liu, B. Bennett, N. Suzuki, Y. Xia, M. Karin, Signaling by proinflammatory cytokines: oligomerization of TRAF2, TRAF6 is sufficient for JNK, IKK activation and target gene induction via an amino-terminal effector domain, Genes Dev. 13 (1999) 1297-1308.

[31] G. Chen, D.V. Goeddel, TNF-R1 signaling: a beautiful pathway, Science 296 (2002) 1634-1635.

[32] A. Devin, A. Cook, Y. Lin, Y. Rodriguez, M. Kelliher, Z. Liu, The distinct roles of TRAF2, RIP in IKK activation by TNF-R1: TRAF2 recruits IKK to TNF-R1 while RIP mediates IKK activation, Immunity 12 (2000) 419-429. 